\title{
Identification of the Thoracic Duct using Indocyanine Green During Cervical Lymphadenectomy
}

\author{
Jeffery Chakedis, MD¹, Lawrence A. Shirley, MD¹, Alicia M. Terando, MD¹, Roman Skoracki, \\ MD'2, and John E. Phay, MD ${ }^{1}$ \\ ${ }^{1}$ Division of Surgical Oncology, Department of Surgery, The Ohio State University Wexner \\ Medical Center and James Cancer Hospital and Solove Research Institute, Columbus, Ohio, USA \\ ${ }^{2}$ Division of Oncologic Plastic Surgery, Department of Plastic Surgery, The Ohio State University \\ Wexner Medical Center and James Cancer Hospital and Solove Research Institute, Columbus, \\ Ohio, USA
}

\begin{abstract}
Background: Injury to the thoracic duct (TD) is the most common complication following a left lateral neck dissection and carries a high degree of morbidity. Currently no routine diagnostic imaging is used to assist with TD identification intra-operatively. Here we present the first clinical experience with lymphangiography using Indocyanine Green (ICG) during lateral neck dissections.

Methods: In six patients undergoing left lateral neck dissection (level II-IV) for either thyroid cancer or melanoma $2.5-5 \mathrm{mg}$ of ICG was injected in the dorsum of the left foot 15 minutes before imaging. Intraoperative imaging was performed with a hand-held Near InfraRed (NIR) camera (Hamamatsu PDE-Neo).
\end{abstract}

Results: In five patients the TD was visualized using NIR fluorescence with time from injection to identification of 15 to 90 minutes. Imaging was optimized by positioning the camera at the angle of the mandible and pointing into the space below the clavicle. There were no adverse reactions from ICG injection; and the time required for imaging was 5-10 minutes. No intraoperative TD injury was identified and no chyle leak occurred post-operatively. In the one patient in whom the TD was not identified it is unclear if it was related to the timing of the injection or duct obliteration from prior dissection.

Conclusion: This is the first described application of ICG lymphangiography for identification of the thoracic duct during left lateral neck dissection. TD identification with ICG is technically feasible simple to perform with NIR imaging and safe making it a potential important adjunct for the surgeon.

Corresponding Author: John E. Phay, MD, 410 W 10th Ave, N908 Doan Hall, Columbus, OH-43210-1267, Phone:(614) 293-7171,

Fax: (614) 293-3465, John.Phay@ osumc.edu.

Authorship:

Study Design: JC, RS, JEP

Data Acquisition: JC, LAS, AMT, JEP

Data Analysis/Interpretation: JC, JEP

Manuscript Preparation/Approval: JC, LAS, AMT, RS, JEP

Conflicts of Interest: We have no conflicts of interest to disclose. 


\section{Keywords}

thoracic duct; near-infrared; indocyanine green; lymphangiography; neck dissection

\section{Introduction:}

Chyle leak or fistula after neck operation occurs in $1-5.4 \%$ of patients with the majority occurring on the left ${ }^{1}$. Injury occurs when the insertion of the thoracic duct (TD) or a branch of is disrupted from the junction of the internal jugular and subclavian vein. The TD is a $3-5$ mm lymphatic channel which normally returns $2-4 \mathrm{~L}$ of triglyceride rich chyle from the thoracic and abdominal compartment to the central venous circulation. There is significant variation in cervical course of the TD however the majority of cadaveric studies have found a single duct and single termination ${ }^{2}$. The right-sided lymphatic duct drains chyle from the head thorax and right arm into the right subclavian vein. Out of all thoracic duct injuries causing fistula up to $25 \%$ occur in the right neck. Thyroidectomy involving a central neck dissection has a low rate of injury and fistula at $1.4 \%^{3}$. Injury has also been reported after penetrating neck trauma or cervical rib resection for thoracic outlet obstruction typically causing chylothorax ${ }^{4}$.

Injury to the TD occurs during neck dissection (modified radical or selective) around the confluence of the internal jugular and subclavian vein (level IV) in the lower neck. Preservation does not always occur as identification can be difficult due to the duct's small and paper-thin appearance as well as its variable anatomic course. Its location and appearance may also be altered by pathologic lymphadenopathy. Injury is frequently technical in nature though patient specific factors which make it difficult to visualize structures properly such as a scarring from a prior surgery or radiation or a short obese neck may impact injury rates. Its fragile nature also contributes to it high inadvertent injury rate. A leak of typically clear chylous fluid can be difficult to detect intraoperatively making identification and repair problematic. Finally the fragile composition of the wall of the thoracic duct often makes repair challenging even when injury is identified.

Morbidity associated from chyle leak can be quite high including wound complications and progression of fluid drainage into the mediastinum and chest resulting in chylothorax. Standard management initially consists of conservative measures including no-fat diet or a diet with only medium chain fatty acids and the use of octreotide ${ }^{5,6}$. If the fistula persists then nothing by mouth and intravenous nutrition can sometimes achieve spontaneous closure within 1 week $^{7}$. A high output chylous leak beyond 5-7 days can cause severe nutritional and immunologic depletion and surgical repair is recommended ${ }^{2}$. Currently a variety of minimally invasive lymphangiography guided procedures can be first attempted instead of open repair and includes ligation of the TD in the chest ${ }^{8}$.

Strategies to recognize and prevent injury to the TD intra-operatively are not commonly used and are sparsely reported in the literature. Indocyanine green (ICG) is a fluorescent dye that binds to intravascular and intra-lymphatic proteins and its fluorescent properties make these vessels visible with near-infrared light ${ }^{9}$. Clinical use of ICG dates back to the 1960's and is frequently used in surgery for a variety of purposes: sentinel lymph node 
identification in gynecological and head and neck cancers evaluation of anastomotic perfusion in colorectal surgery fluorescent guided resection of liver tumors and lymphatic mapping during surgical correction of lymphedema ${ }^{10-14}$. ICG has been used successfully in case reports to identify the thoracic duct in the abdomen and chest to allow for ligation in the treatment of post-operative chylothorax and chylous ascites ${ }^{15-17}$.

Here we present the first use of a technique to identify and preserve the thoracic duct during lateral neck dissection using ICG lymphangiography. We have determined that identification of the thoracic duct is technically feasible by applying near-infrared (NIR) imaging systems that are routinely used. The clinical application of this technique will require subsequent validation to prove improvement in rates of chylous fistula and intra-operative recognition of injury with a decrease in subsequent morbidity.

\section{Methods:}

\section{Patient Cohort}

All patients were recruited from the surgical oncology clinic at a single institution from May to September of 2017. Patients in whom a left lateral neck dissection was indicated in conjunction with other procedures were included. ICG is contraindicated for minors breast feeding patients and those with allergies to iodine and was not used in these patients. This study was approved by the institutional review board. The imaging device and the ICG compound are FDA approved and used routinely in clinical care.

\section{Surgical Technique and post-operative care}

A left lateral dissection (LND) was performed with no deviations in the technical aspects of the procedure for the purposes of this study by 3 surgical oncologists (LAS AMT JEP). Dissection in the neck included levels II III and IV routinely with level V and VI dissection if indicated. The internal jugular vein spinal accessory nerve recurrent laryngeal and vagus nerve were not resected unless involved with tumor. Approximately 15 minutes before dissection of level IV the dorsum of the left foot just below the ankle was injected with ICG subcutaneously. Imaging was performed periodically throughout dissection of level IV as the subclavian vein and junction with the internal jugular vein was performed. Time from injection of ICG to imaging of thoracic duct was recorded as well as total imaging time. After the dissection was completed with removal of the specimen and the thoracic duct identified final imaging was performed. A surgical drain was used at the completion of the operation and placed to bulb suction.

Indocyanine green was reconstituted in standard vials of $25 \mathrm{mg}$ of powder with $10 \mathrm{~mL}$ of sterile saline to a concentration of $2.5 \mathrm{mg} / \mathrm{mL}$. A $25 \mathrm{~g}$ insulin type needle and $5 \mathrm{~mL}$ syringe was utilized for injection subcutaneously. Identification of an area of normal skin free from prior injury or infection was used on the dorsum of the foot. Alcohol was used to clean the skin and 1-2 $\mathrm{mL}(2.5-5 \mathrm{mg})$ of ICG was used for each injection. If the thoracic duct was not identified after 30 minutes time from injection the ICG was re-dosed with another 1-2 mL and only one re-dosing was used. After the operation patients were admitted to the surgical ward for monitoring. A high fat diet was started on post-operative day 1 to evaluate for 
chylous fistula. Drain output was monitored for chyle after starting a diet and if no evidence of leak the patient was discharged on post-operative day 2 after removal of the drain.

\section{Imaging Procedures}

Images were obtained during surgery at a time of the surgeons discretion when identification of the thoracic duct is optimal. Fluorescence images were obtained using the PDE-neo II imager (Hamamatsu model C10935-400 Hamamatsu City Japan). The camera is a handheld device draped in a sterile covering and used to obtain images during the surgery which are seen on an adjacent monitor. The operating room lights are turned off during imaging. Imaging is performed with the probe held at the patients head and pointed caudally and medially into the space below the clavicle Figure 1. This provides the optimal angle for imaging into this narrow space. Image collection is real-time and video as well as select images are saved on a USB port within the device. Imaging during identification of the thoracic duct lasted 1-2 minutes. Final imaging of the thoracic duct after completion of the dissection lasted up to 5 minutes. After the operation all images were transferred to a protected network drive.

\section{Results:}

Imaging of the thoracic duct was attempted on six patients and successful in five (83.3\%) Table 1. Indications for operation included thyroid cancer (medullary poorly differentiated and papillary) and melanoma. None of the patients had a history of neck radiation however three had a history of prior neck surgery. One patient underwent revision lateral neck dissection for cancer recurrence and two patients had recent central neck surgery. Overall the time from injection to NIR imaging and identification ranged from 15-90 minutes. The time varied between patients significantly due to trial and error as well as uncertainty in the time it would take ICG to travel from the legs to the neck. There was no standard time that NIR imaging was used and the area where the thoracic duct should be was imaged periodically throughout the dissection. In the patient that took 90 minutes from injection the ICG was injected before starting the neck dissection and only imaged after it was completed. In three patients ICG was re-dosed to assist with identification.

Time to perform imaging in total was 5-10 minutes per patient and was split between multiple imaging sessions. This time was added to the operation by performing the procedure and included turning off the operating room lights using the NIR imager and recording video or taking appropriate pictures. No patients had an identified injury to the thoracic duct intra-operatively or post-operative chyle leak. There were no surgical complications were related to ICG injection or imaging and there were no adverse events with subcutaneous injection of ICG.

The TD was identified in the five patients during the dissection around the level of the subclavian vein (SV) in its junction with the internal jugular vein (IJV). In patient \#4 a structure that appeared to be the TD was identified during dissection without use of NIR imaging. A branch from this structure was clipped as it entered the specimen and then imaging confirmed this was in fact the TD Figure 2. The TD was clearly visualized after completing the dissection and could be imaged terminating in the junction between the SC 
and IJV Supplemental video 1 . In patient \#5 the TD was identified by fluorescence NIR imaging alone and further dissected after identification Figure 3. A level IV lymph node was also weakly fluorescent in this patient. Fluorescent chylous fluid containing bubbles could be seen entering the SV Supplemental video \#2. Fluorescence was only seen in the TD and lymph nodes and not in the vascular structures.

In one patient the thoracic duct was not identified due to unknown factors. This patient underwent resection of a large left thyroid mass at another hospital 1 month prior to the current operation. In the current operation he had a sternotomy resection of substernal mass with en bloc sternectomy and resection of recurrent laryngeal nerve by the thoracic oncologist before performance of the lateral neck dissection. ICG re-dosing was performed and ample time was given for imaging.

\section{Discussion:}

We present the first described application of ICG and NIR imaging system to identify the thoracic duct in the neck during a left lateral neck dissection. This concept was initially born out of management of the first patient in our series with MEN2B undergoing revision bilateral neck dissection for recurrence in level IV. We were concerned about dissection in this area due to scar obliterating the planes and potential injury to the TD as there is a higher rate of chylous fistula in patients with prior neck dissection or radiation ${ }^{6}$. The technique was adapted from reports of ICG identifying sentinel lymph nodes related to breast melanoma and cervical cancer ${ }^{9}$. Ultimately the technique was successful in this first patient and later confirmed with the subsequent patients with an overall identification rate of $83.3 \%$.

ICG has been used to assess blood flow tissue viability and to identify lymphatic tissues since the 1950's and its use is now prevalent in cancer surgery ${ }^{9}$. Multiple NIR imaging devices have been FDA approved beginning in 2005 with the SPY system by Novadaq Technologies ${ }^{18}$. These systems primarily use ICG as their imaging fluorophore which is reimbursable for certain procedures. Lymphangiography using ICG and NIR imaging is commonly used in patients with lymphedema to identify the blocked lymphatic channels for surgical reconstruction ${ }^{19}$. Our described technique is an extension of these concepts and a new application of the existing technology that requires continued refinement. As our experience with NIR imaging grows the accuracy of our dissection and identification rate will likely improve. A learning curve of 20 laparoscopic ICG directed SLN procedures was required to increase accuracy in one study ${ }^{20}$. We are currently performing a clinical trial to optimize and standardize the technique of combining ICG directed identification of the thoracic duct with lateral neck dissection.

There have been few studies reported in the literature which have used ICG to image the TD and none have been in the neck. Animal models of this process have included ICG lymphangiography of the TD in the thoracic cavity in rats and pigs ${ }^{21}$. The TD was able to be imaged within 5 minutes of injection in the lower leg and the fluorescence activity lasted for 60 minutes. In humans identification of the TD using ICG has been performed previously in the chest and abdomen in case reports. After injury to the TD in the chest or abdomen during esophagectomy with resultant chylothorax or chylous ascites ICG injection in the lower leg 
resulted in identification of the leak to ligate it succefully ${ }^{15,16}$. The optimal time from injection of ICG to imaging in humans still needs to be determined though it appears the florescence signal lasts for up to 90 minutes. Further optimization of the technique will involve early injection of ICG before dissection and then subsequent imaging of the thoracic duct area before during and after dissection is performed.

Though none of our patients had a TD injury or chylous fistula the technique may be helpful in identifying TD injury. Currently there are no routinely used diagnostics available to identify or treat a chyle leak intra-operatively. A single study using abdominal compression after neck dissection revealed unrecognized chyle leak in $6.3 \%$ of patients ${ }^{22}$. Two out of 13 patients had persistent leak after identifying and suture ligating the newly identified fistula and had persistent leaks post-operatively. When high output chyle leak is recognized postoperatively and fails conservative management there are reports of minimally invasive radiologic procedures using embolization or sclerosing agents ${ }^{23}$. Bipedal lymphangiography using ethiodized oil injected into the lymphatic channels in the dorsum of the foot has been used to identify the abdominal and thoracic portions of the duct and identify leak in the chest ${ }^{24}$. However the majority of chylous fistulas require open surgical ligation and this novel application of NIR imaging may be effective in identification of the TD to assist with fistula closure.

The technique of NIR imaging routinely during neck dissection is limited by several issues which are common to this technology. Fluorescence penetration through tissues is low at $8 \mathrm{~mm}$ maximal depth ${ }^{25}$. Significant dissection is required in the tissue before fluorescence can be seen. In our experience the anatomy is delineated after dissection and it is a dynamic process which requires switching to the hand-held imager intermittently during the dissection. The whole process can take time and in our series added 5-10 minutes of general anesthesia. Furthermore cost can be significant though ICG is inexpensive an investment in the imaging equipment is a required. Optimization and standardization of the injection and imaging technique is required before this method can be adopted and utilized to identify or prevent thoracic duct injury.

In conclusion we present the first application of ICG and NIR imaging for purpose of identifying the TD in the neck during lateral neck dissection. This technique is feasible simple to perform and safe making it a potential important tool for the surgeon. Given that chyle leaks are the most common complication after a left lateral neck dissection this technique has the potential to change the current practice of this operation. The utility of the technique will certainly be helpful in identifying the thoracic duct in select patients in which dissection is difficult such as re-operative cases prior neck radiation or trauma to the area. It will also have utility in identifying the site of injury when chylous fluid is identified in the wound intra-operatively and confirm that repair is complete. Further work is required to optimize the procedure so that it can be easily incorporated into routine practice.

\section{Supplementary Material}

Refer to Web version on PubMed Central for supplementary material. 


\title{
Acknowledgments
}

Grant Support: None

\author{
Abbreviations: \\ TD \\ Thoracic Duct \\ ICG IndoCyanine Green \\ LND Lateral Neck Dissection \\ NIR Near-InfraRed \\ (SV) Subclavian Vein \\ (IJV) Internal Jugular Vein \\ MEN Multiple endocrine neoplasia \\ PTC Papillary thyroid cancer
}

\section{References:}

1. de Gier HH, Balm AJ, Bruning PF, Gregor RT, Hilgers FJ. Systematic approach to the treatment of chylous leakage after neck dissection. Head Neck 1996;18(4):347-351. [PubMed: 8780946]

2. Scorza LB, Goldstein BJ, Mahraj RP. Modern management of chylous leak following head and neck surgery: a discussion of percutaneous lymphangiography-guided cannulation and embolization of the thoracic duct. Otolaryngol Clin North Am 2008;41(6):1231-1240 xi. [PubMed: 19040982]

3. Roh JL, Yoon YH, Park CI. Chyle leakage in patients undergoing thyroidectomy plus central neck dissection for differentiated papillary thyroid carcinoma. Ann Surg Oncol 2008;15(9):2576-2580. [PubMed: 18592317]

4. Nussenbaum B, Liu JH, Sinard RJ. Systematic management of chyle fistula: the Southwestern experience and review of the literature. Otolaryngol Head Neck Surg 2000;122(1):31-38. [PubMed: 10629479]

5. Brennan PA, Blythe JN, Herd MK, Habib A, Anand R. The contemporary management of chyle leak following cervical thoracic duct damage. Br J Oral Maxillofac Surg 2012;50(3):197-201. [PubMed: 21376436]

6. Swanson MS, Hudson RL, Bhandari N, Sinha UK, Maceri DR, Kokot N. Use of Octreotide for the Management of Chyle Fistula Following Neck Dissection. JAMA Otolaryngol Head Neck Surg 2015;141(8):723-727. [PubMed: 26135979]

7. Smoke A, Delegge MH. Chyle leaks: consensus on management? Nutr Clin Pract 2008;23(5):529_ 532. [PubMed: 18849558]

8. Campisi CC, Boccardo F, Piazza C, Campisi C. Evolution of chylous fistula management after neck dissection. Curr Opin Otolaryngol Head Neck Surg 2013;21(2):150-156. [PubMed: 23449286]

9. Zelken JA, Tufaro AP. Current Trends and Emerging Future of Indocyanine Green Usage in Surgery and Oncology: An Update. Ann Surg Oncol 2015;22 Suppl 3:S1271-1283. [PubMed: 26193966]

10. Chang DW, Suami H, Skoracki R. A prospective analysis of 100 consecutive lymphovenous bypass cases for treatment of extremity lymphedema. Plast Reconstr Surg 2013;132(5):1305-1314. [PubMed: 24165613]

11. Araki K, Mizokami D, Tomifuji M et al. Novel Indocyanine Green-Phytate Colloid Technique for Sentinel Node Detection in Head and Neck: Mouse Study. Otolaryngol Head Neck Surg 2014;151(2):279-285. [PubMed: 24732687] 
12. Boni L, David G, Dionigi G, Rausei S, Cassinotti E, Fingerhut A. Indocyanine green-enhanced fluorescence to assess bowel perfusion during laparoscopic colorectal resection. Surg Endosc 2016;30(7):2736-2742. [PubMed: 26487209]

13. van der Vorst JR, Schaafsma BE, Hutteman M et al. Near-infrared fluorescence-guided resection of colorectal liver metastases. Cancer 2013;119(18):3411-3418. [PubMed: 23794086]

14. Papadia A, Imboden S, Siegenthaler F et al. Laparoscopic Indocyanine Green Sentinel Lymph Node Mapping in Endometrial Cancer. Ann Surg Oncol 2016;23(7):2206-2211. [PubMed: 26790667]

15. Kamiya K, Unno N, Konno H. Intraoperative indocyanine green fluorescence lymphography a novel imaging technique to detect a chyle fistula after an esophagectomy: report of a case. Surg Today 2009;39(5):421-426. [PubMed: 19408081]

16. Matsutani T, Hirakata A, Nomura T et al. Transabdominal approach for chylorrhea after esophagectomy by using fluorescence navigation with indocyanine green. Case Rep Surg 2014;2014:464017. [PubMed: 25105050]

17. Papadia A, Imboden S, Mohr S, Lanz S, Nirgianakis K, Mueller MD. Indocyanine Green Fluorescence Imaging in the Surgical Management of an Iatrogenic Lymphatic Fistula: Description of a Surgical Technique. J Minim Invasive Gynecol 2015;22(7):1304-1306. [PubMed: 26122899]

18. AV DS, Lin H, Henderson ER, Samkoe KS, Pogue BW. Review of fluorescence guided surgery systems: identification of key performance capabilities beyond indocyanine green imaging. Journal of biomedical optics 2016;21(8):80901. [PubMed: 27533438]

19. Ogata F, Narushima M, Mihara M, Azuma R, Morimoto Y, Koshima I. Intraoperative lymphography using indocyanine green dye for near-infrared fluorescence labeling in lymphedema. Ann Plast Surg 2007;59(2):180-184. [PubMed: 17667413]

20. Papadia A, Imboden S, Gasparri ML, Siegenthaler F, Fink A, Mueller MD. Endometrial and cervical cancer patients with multiple sentinel lymph nodes at laparoscopic ICG mapping: How many are enough? J Cancer Res Clin Oncol 2016;142(8):1831-1836. [PubMed: 27318493]

21. Ashitate Y, Tanaka E, Stockdale A, Choi HS, Frangioni JV. Near-infrared fluorescence imaging of thoracic duct anatomy and function in open surgery and video-assisted thoracic surgery. $\mathrm{J}$ Thorac Cardiovasc Surg 2011;142(1):31-38.e31-32. [PubMed: 21477818]

22. Cernea CR, Hojaij FC, De Carlucci D, Jr. et al. Abdominal compression: a new intraoperative maneuver to detect chyle fistulas during left neck dissections that include level IV. Head Neck 2012;34(11):1570-1573. [PubMed: 22290583]

23. Itkin M, Kucharczuk JC, Kwak A, Trerotola SO, Kaiser LR. Nonoperative thoracic duct embolization for traumatic thoracic duct leak: experience in 109 patients. J Thorac Cardiovasc Surg 2010;139(3):584-589; discussion 589-590. [PubMed: 20042200]

24. Sachs PB, Zelch MG, Rice TW, Geisinger MA, Risius B, Lammert GK. Diagnosis and localization of laceration of the thoracic duct: usefulness of lymphangiography and CT. AJR Am J Roentgenol 1991;157(4):703-705. [PubMed: 1892021]

25. Schaafsma BE, Mieog JS, Hutteman M et al. The clinical use of indocyanine green as a nearinfrared fluorescent contrast agent for image-guided oncologic surgery. J Surg Oncol 2011;104(3): 323-332. [PubMed: 21495033] 


\section{SYNOPSIS:}

This is the first report of using Indocyanine Green lymphangiography in six patients to identify the thoracic duct during lateral neck dissection. The technique may be a useful tool as it is technically feasible safe and simple to perform. 


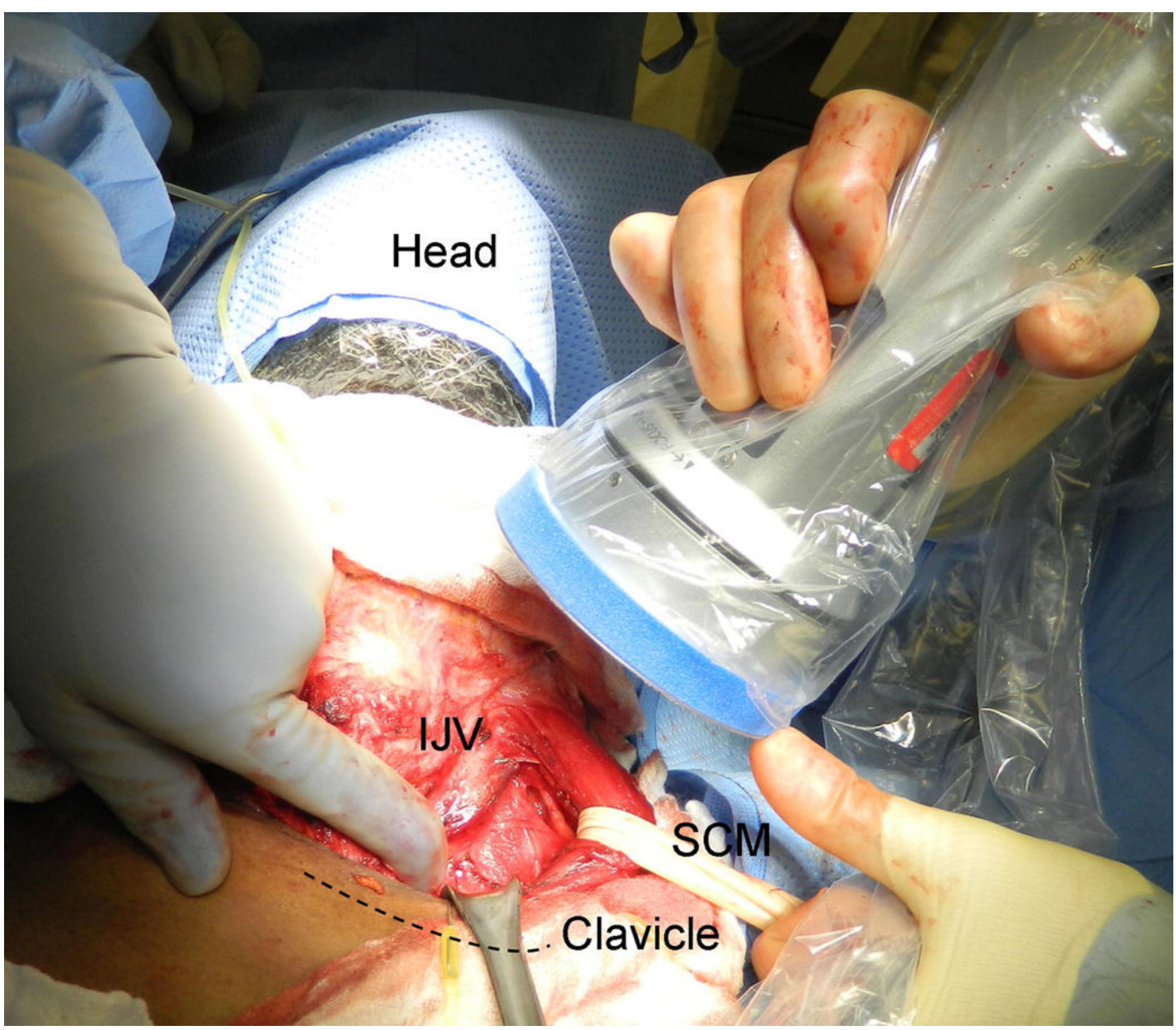

Figure 1.

Position for imaging the thoracic duct. The probe is held at the patients head near the angle of the mandible and pointed caudally to image the space beneath the clavicle. The sternocleidomastoid muscle (SCM) soft tissue above the clavicle (dashed line), and internal jugular vein (IJV) are retracted to allow for better visualization. 


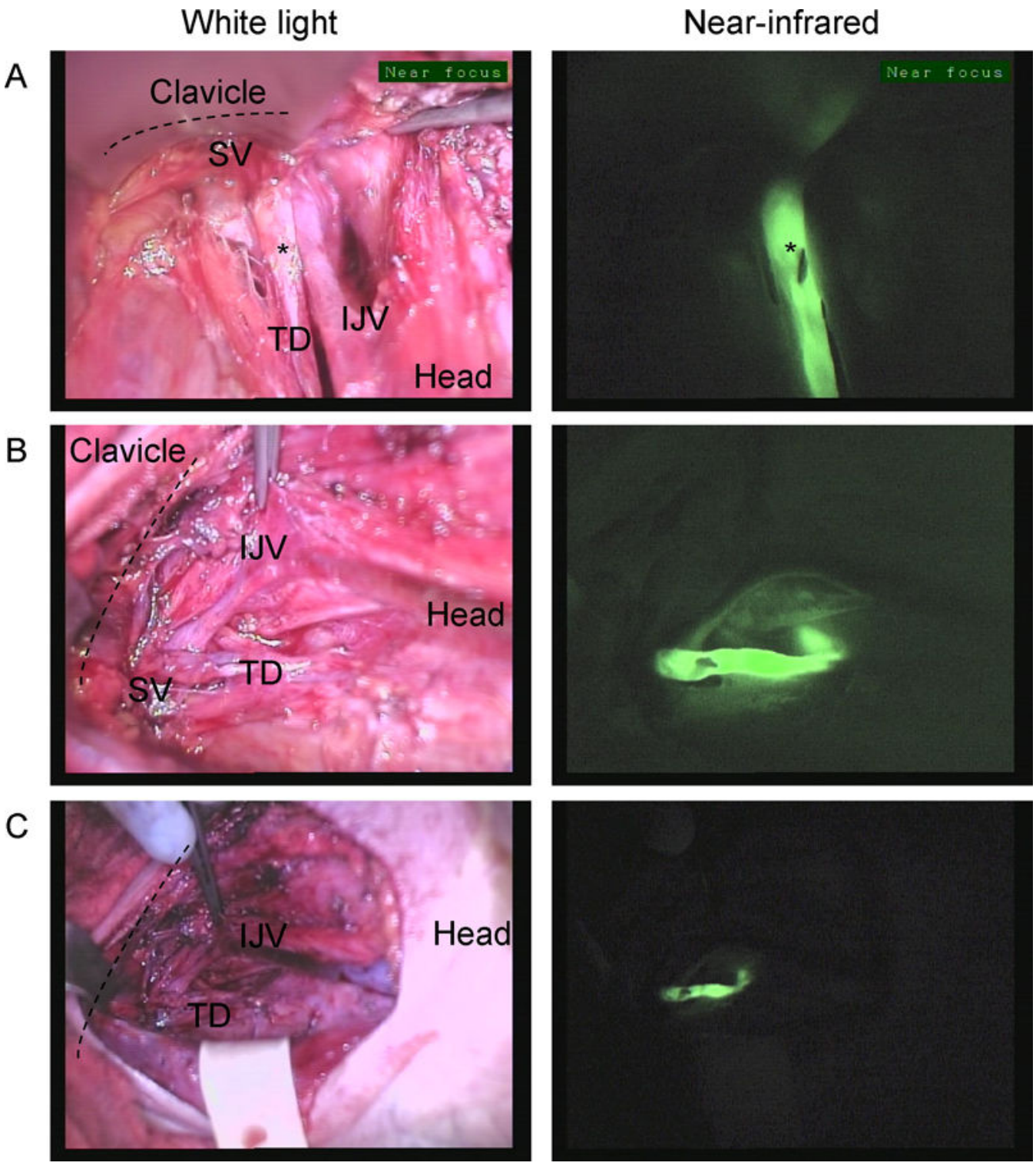

Figure 2.

Near-infrared imaging of the thoracic duct in Patient \#4. The thoracic duct (TD) inserting on the junction between the Internal jugular vein (IJV) and Subclavian vein (SV) from 3 different points of view. The TD is marked with a clip $(*)$ which is clearly visible on the near-infrared images A. Imaging from the angle of the mandible down to the space below the clavicle (dashed line), B. Imaging as the operator is standing on the left side of the patient C. Similar view as in B. but imaged from a farther distance from the patient. 

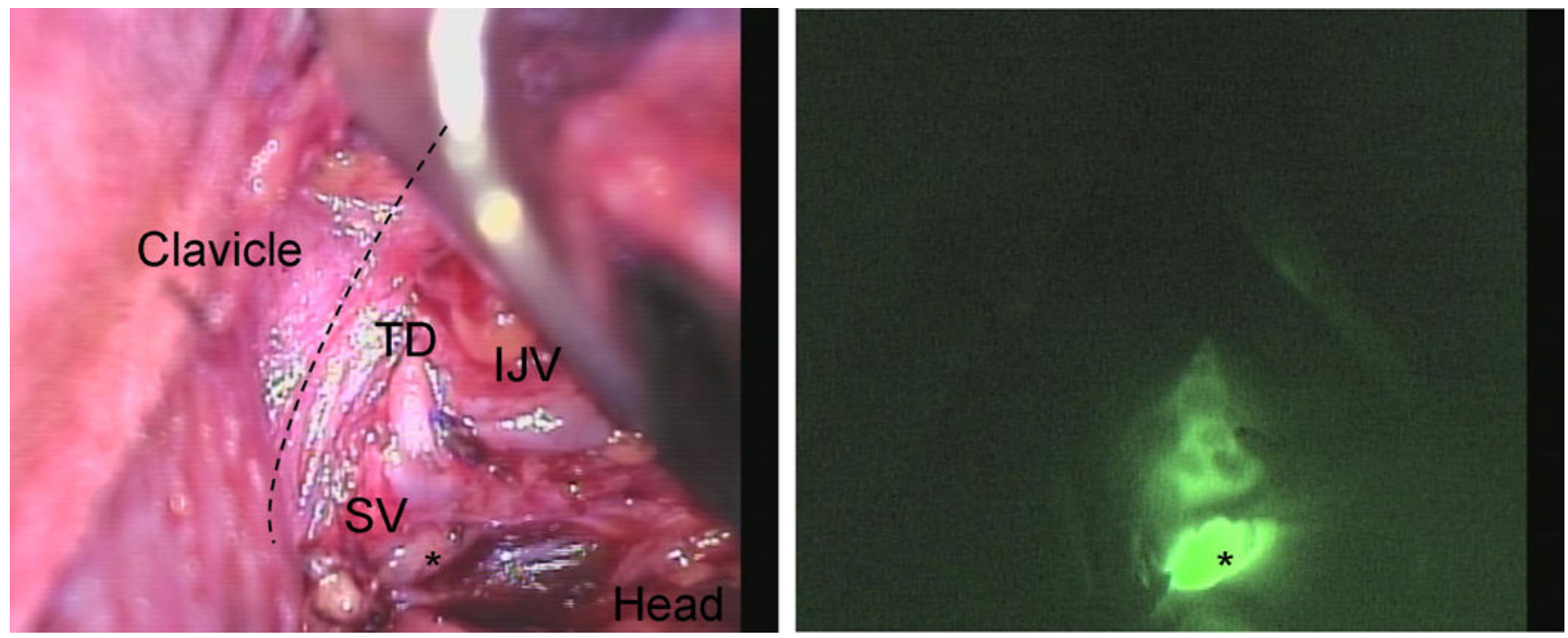

Figure 3.

Near-infrared imaging of the thoracic duct in Patient \#5. The thoracic duct (TD) inserting on the junction between the Internal jugular vein (IJV) and Subclavian vein (SV) imaging from the head down to the space below the clavicle. A level IV lymph node (*) is also fluorescent. 


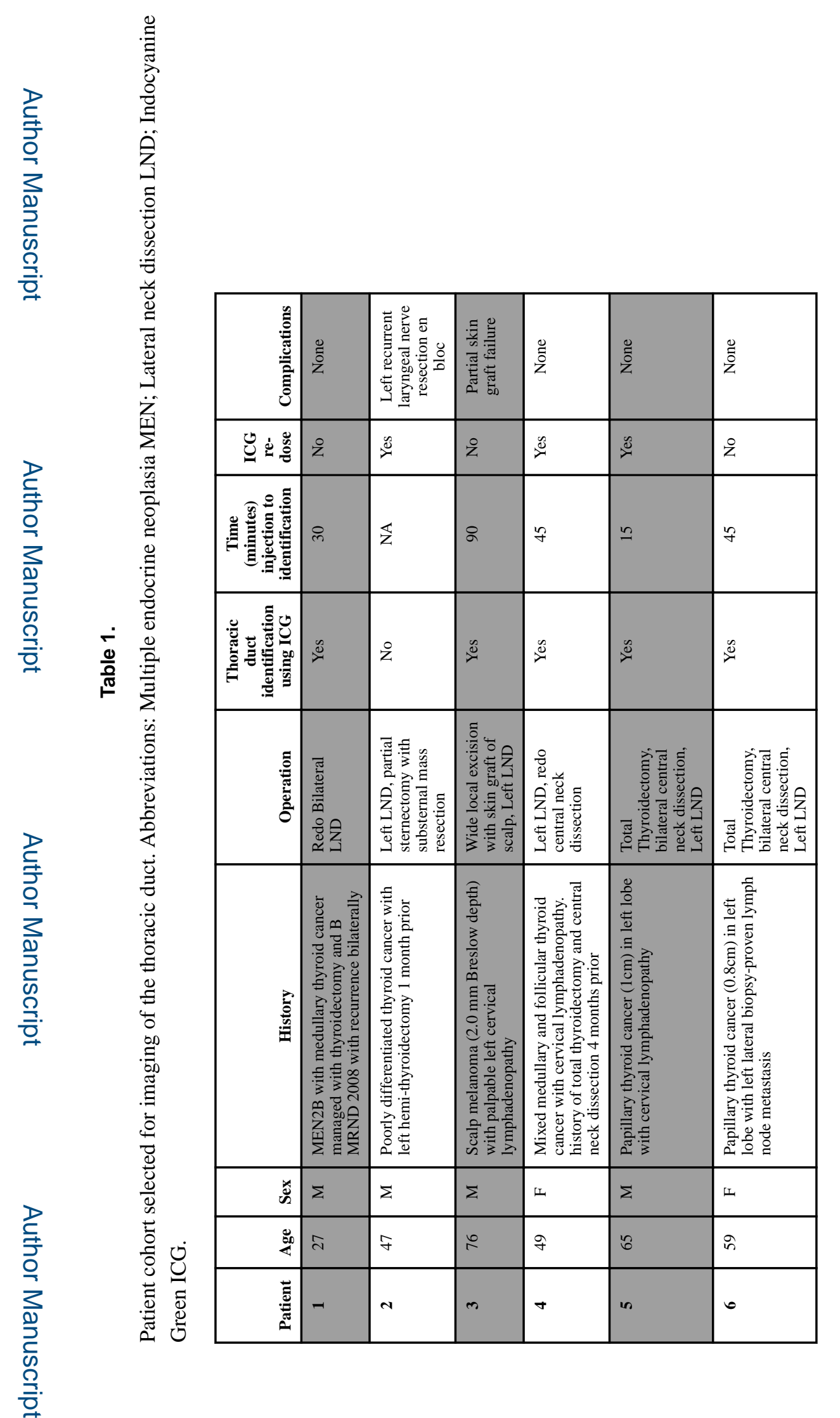

Ann Surg Oncol. Author manuscript; available in PMC 2019 November 01. 